\title{
Gull Alpha Power of the Ampadu-Type: Properties and Applications
}

\author{
Clement Boateng Ampadu
}

31 Carrolton Road, Boston, MA 02132-6303, USA

e-mail: drampadu@hotmail.com

\begin{abstract}
This paper introduces a new statistical distribution called Gull Alpha Power of the Ampadu Type (GAPA- $G$ for short). The new distribution is inspired by the Gull Alpha Power of [1] and the Ampadu-G of [2]. Some properties with application are investigated.
\end{abstract}

\section{Introduction}

A random variable $X$ is said to follow the Gull Alpha Power family of distributions if its $\mathrm{CDF}$ is given by

$$
G(x ; \alpha, \xi)=\frac{\alpha F(x ; \xi)}{\alpha^{F(x ; \xi)}} \quad[1]
$$

where $\alpha>1, \xi$ is a vector of parameters in the baseline distribution, and $x \in$ $\operatorname{Supp}(F)$. Obviously, the PDF can be obtained by differentiating the CDF. On the other hand a random variable $J$ is said to follow the Ampadu-G family of distributions if its CDF is given by

$$
K(x ; \lambda, \omega)=\frac{1-e^{-\lambda G(x ; \omega)^{2}}}{1-e^{-\lambda}} \quad[2]
$$

where $\lambda \in \mathbb{R} \backslash\{0\}, x \in \operatorname{Supp}(G)$, and $\omega$ is a vector of parameters in the baseline distribution. By a modification of Ampadu-G, this paper introduces a new kind of

Received: December 1, 2020; Accepted: January 16, 2021

2010 Mathematics Subject Classification: 62Exx.

Keywords and phrases: Gull Alpha Power, Ampadu-G, Alpha Power Transform.

Copyright (c) 2021 the Author 
Gull Alpha Power distribution and studies some of its properties with applications. We should note that the studies generalizing the Alpha Powe Transform [3] have appeared in the literature [4]-[16], for example.

\section{Organization of Manuscript}

This manuscript is organized as follows. In Section 3, the CDF and PDF of the new family is presented. A sub-model of the new family is discussed in Section 4, there the CDF, PDF, hazard function (HF), and survival function (SF) are visualized. In Section 5, we derive some mathematical properties of the new family, including quantile function, random number generation, $r$ th non-central moments, and the Renyi entropy. In Section 6, the method of maximum likelihood in estimating model parameters are discussed. In Section 7, a Monte Carlo simulation study is carried out, it is shown that the method of maximum likelihood is adequate in estimating model parameters. In Section 8, we demonstrate usefulness of the new family to a data set coming from the material engineering sciences. Section 9 , presents a characterization of the new family in terms of the hazard function. In Section 10, we propose some further research directions.

\section{The New Family Defined}

\subsection{The CDF of the New Family}

The CDF of GAPA- $G$ is given by

$$
F(x ; \alpha, \xi)=\frac{\left(1+e^{-\alpha}\right) G(x ; \xi)}{1+e^{-\alpha G(x ; \xi)}}
$$

where $\alpha \in \mathbb{R}, G$ is a baseline $\mathrm{CDF}, x \in \operatorname{Supp}(G)$ and the support of $\xi$ depends on the chosen baseline distribution.

\subsection{The PDF of the New Family}

The PDF of GAPA- $G$ is given by 


$$
f(x ; \alpha, \xi)=\frac{g(x ; \xi)\left(e^{\alpha}+1\right) e^{\alpha(G(x ; \xi)-1)}\left(\alpha G(x ; \xi)+e^{\alpha G(x ; \xi)}+1\right)}{\left(e^{\alpha G(x ; \xi)}+1\right)^{2}}
$$

where $\alpha \in \mathbb{R}, G$ is a baseline CDF with $\operatorname{PDF} g, x \in \operatorname{Supp}(G, g)$ and the support of $\xi$ depends on the chosen baseline distribution.

\section{A Sub-Model of the New Family}

The PDF of the sub-model is given by

$$
=\frac{\left(e^{c}+1\right) e^{c\left(\frac{1}{e^{\frac{a-x}{b}}+1}-1\right)+\frac{a+x}{b}}\left(e^{a / b}\left(e^{\frac{c}{e^{\frac{a-x}{b}}+1}}+1\right)+e^{x / b}\left(e^{\frac{c}{\frac{a-x}{b}}+1}+c+1\right)\right)}{b\left(e^{a / b}+e^{x / b}\right)^{3}\left(e^{\frac{c}{e^{\frac{a-x}{b}}}+1}+1\right)^{2}}
$$

and the CDF is given by

$$
F(x ; a, b, c)=\frac{e^{-c}+1}{\left(e^{-\frac{x-a}{b}}+1\right)\left(e^{-\frac{c}{e^{-\frac{x-a}{b}}+1}}+1\right)}
$$

where $x, a, c \in \mathbb{R}$, and $b>0$. We call the distribution with above PDF and CDF the Gull Alpha Power Ampadu-Logisitic family. We write

$$
J \sim G A P A L(a, b, c)
$$

if $J$ is a Gull Alpha Power Ampadu-Logistic random variable. 


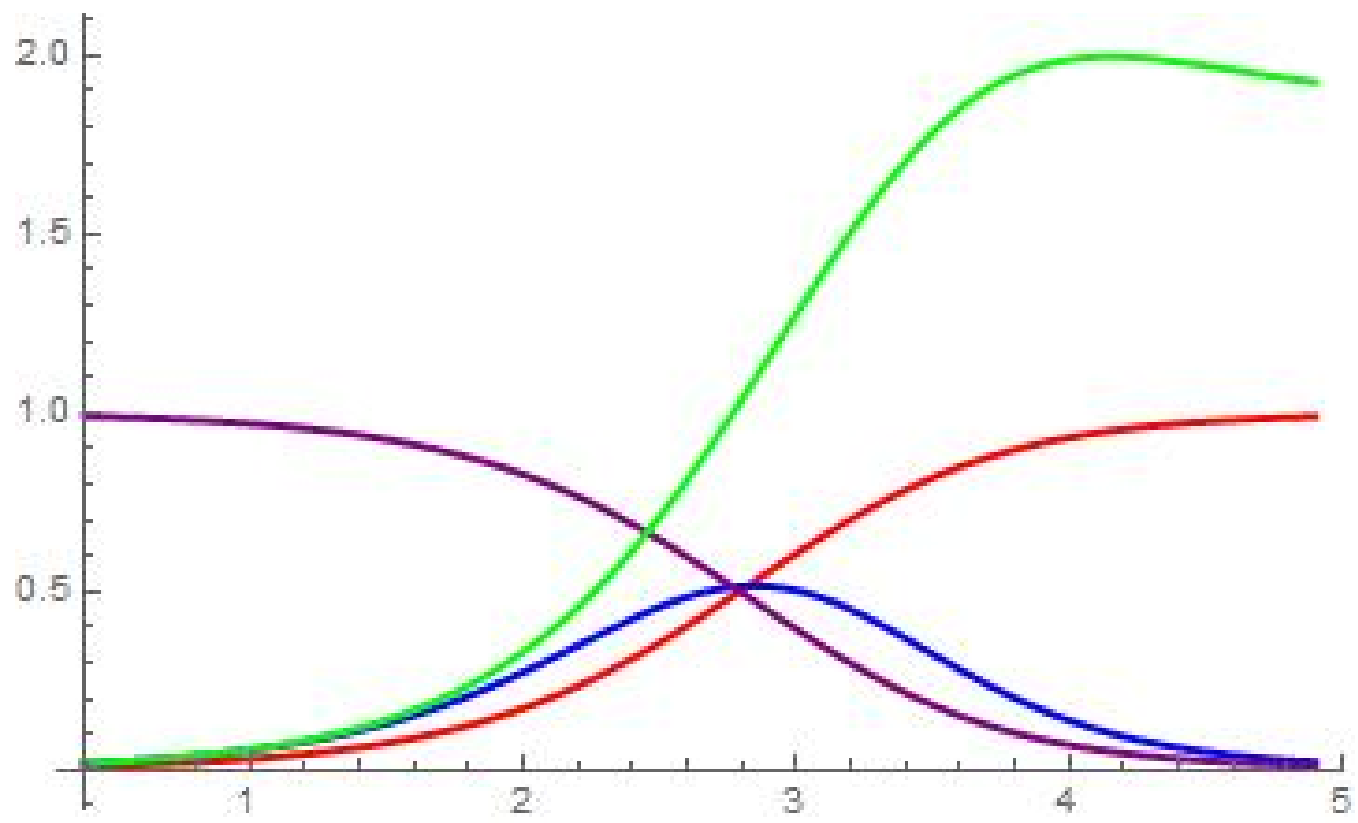

Figure 1: The CDF (red), PDF(blue), SF(purple), and HF (green) of GAPAL(3.2081,0.541169,-1.01248).

\section{Some Mathematical Properties}

\subsection{Quantile Function}

Theorem 5.1. The quantile function of $G A P A-G$ is given by

$$
Q(x)=G^{-1}\left(\frac{W\left(\frac{\alpha x e^{\alpha-\frac{e^{\alpha} \alpha x}{e^{\alpha}+1}}}{e^{\alpha}+1}\right)}{\alpha}+\frac{e^{\alpha} x}{e^{\alpha}+1}\right)
$$

where $W(z)$ gives the principal solution for $w$ in $z=w e^{w}, 0<x<1$, and $G^{-1}$ is the quantile of the baseline distribution with CDF G.

Proof. Let $0<x<1$. We must solve the following equation for $Q(x)$

$$
x=\frac{\left(1+e^{-\alpha}\right) G(Q(x))}{1+e^{-\alpha G(Q(x))}} .
$$


Let $y=G(Q(x))$, using computer software like MATHEMATICA one can show that solving for $y$ in the following equation below

$$
x=\frac{\left(1+e^{-\alpha}\right) y}{1+e^{-\alpha y}}
$$

yields

$$
G(Q(x))=\frac{W\left(\frac{\alpha x e^{\alpha-\frac{e^{\alpha} \alpha x}{e^{\alpha}+1}}}{e^{\alpha}+1}\right)}{\alpha}+\frac{e^{\alpha} x}{e^{\alpha}+1} .
$$

Thus

$$
Q(x)=G^{-1}\left(\frac{W\left(\frac{\alpha x e^{\alpha-\frac{e^{\alpha} \alpha x}{e^{\alpha}+1}}}{e^{\alpha}+1}\right)}{\alpha}+\frac{e^{\alpha} x}{e^{\alpha}+1}\right)
$$

where $W(z)$ gives the principal solution for $w$ in $z=w e^{w}$.

Table 1: Some Quantile Values

\begin{tabular}{ccc}
\hline$x$ & $Q(x)$ of $\operatorname{GAPAL}(0.5,0.3,-0.7)$ & $Q(x)$ of $\operatorname{GAPAN}(0.5,0.3,-0.7)$ \\
\hline 0.1 & -0.2854443 & 0.0526973 \\
\hline 0.2 & -0.04575005 & 0.1752752 \\
\hline 0.3 & 0.1119788 & 0.263527 \\
\hline 0.4 & 0.2400855 & 0.3392247 \\
\hline 0.5 & 0.3566874 & 0.4105542 \\
\hline 0.6 & 0.4725202 & 0.4827822 \\
\hline 0.7 & 0.5982301 & 0.5614392 \\
\hline 0.8 & 0.7516267 & 0.6557718 \\
\hline 0.9 & 0.9843711 & 0.7910821 \\
\hline \hline
\end{tabular}




\subsection{Random Number Generation}

Random numbers from GAPA- $G$ can be obtained via

$$
X=G^{-1}\left(\frac{W\left(\frac{\alpha U e^{\alpha-\frac{e^{\alpha} \alpha U}{e}+1}}{e^{\alpha}+1}\right)}{\alpha}+\frac{e^{\alpha} U}{e^{\alpha}+1}\right)
$$

where $W(z)$ gives the principal solution for $w$ in $z=w e^{w}, U \sim \operatorname{Uniform}(0,1)$, and $G^{-1}$ is the quantile of the baseline distribution with CDF $G$.

\section{3 $r$ th Non-Central Moments}

Theorem 5.2. The rth non-central moments of GAPA-G can be expressed as

$$
\mu_{r}^{\prime}=\sum_{i=0}^{\infty} \sum_{k=0}^{i} \sum_{n=k}^{\infty} \sum_{j=0}^{\infty} \sum_{m=0}^{j} \Omega_{i, k, n, j, m} E\left[U^{i-k+n+m}\right]
$$

where $\Omega_{i, k, n, j, m}$ is defined as in the proof of the theorem, $U \sim \operatorname{Uniform}(0,1)$, and $E[\cdot]$ denotes an expectation.

Proof. According to [17], we can write

$$
Q_{X}(u)=\sum_{i=0}^{\infty} h_{i} u^{i}
$$

where the coefficients are suitably chosen real numbers that depend on the parameters of the $G(x)$ distribution. For a power series raised to a positive integer $r \geq 1$, we have

$$
\left(Q_{X}(u)\right)^{r}=\left(\sum_{i=0}^{\infty} h_{i} u_{i}\right)^{r}=\sum_{i=0}^{\infty} \delta_{r, i} u^{i}
$$

where $\delta_{r, i}$ are obtained from $\delta_{r, i}=\left(i h_{0}\right)^{-1} \sum_{s=1}^{i}[s(r+1)-i] h_{s} \delta_{r, i-s}$ with $\delta_{r, 0}=h_{0}^{r}$ for $i=1,2, \cdots[18$. Thus we have the following

$$
\mu_{r}^{\prime}=\sum_{i=0}^{\infty} \delta_{r, i} E\left[\left(\frac{W\left(\frac{\alpha U e^{\alpha-\frac{e^{\alpha} \alpha U}{e^{\alpha}+1}}}{e^{\alpha}+1}\right)}{\alpha}+\frac{e^{\alpha} U}{e^{\alpha}+1}\right)^{i}\right]
$$


where $E(\cdot)$ is an expectation. By the binomial theorem we can write

$$
\left(\frac{W\left(\frac{\alpha U e^{\alpha-\frac{e^{\alpha} \alpha U}{e^{\alpha}+1}}}{e^{\alpha}+1}\right)}{\alpha}+\frac{e^{\alpha} U}{e^{\alpha}+1}\right)^{i}
$$

as

$$
\sum_{k=0}^{i}\left(\begin{array}{l}
i \\
k
\end{array}\right)\left(\frac{e^{\alpha}}{e^{\alpha}+1}\right)^{i-k} U^{i-k} \frac{W\left(\frac{\alpha U e^{\alpha-\frac{e^{\alpha} \alpha U}{e^{\alpha}+1}}}{e^{\alpha}+1}\right)^{k}}{\alpha^{k}} .
$$

Using integer powers of the Lambert $\mathrm{W}$ function, we can write

$$
W\left(\frac{\alpha U e^{\alpha-\frac{e^{\alpha} \alpha U}{e^{\alpha}+1}}}{e^{\alpha}+1}\right)^{k}
$$

as

$$
\sum_{n=k}^{\infty} \frac{-k(-n)^{n-k-1}}{(n-k) !}\left(\frac{\alpha U e^{\alpha-\frac{e^{\alpha} \alpha U}{e^{\alpha}+1}}}{e^{\alpha}+1}\right)^{n} .
$$

By the power series representation for the exponential function, and the binomial theorem, we can write

$$
\left(\frac{\alpha U e^{\alpha-\frac{e^{\alpha} \alpha U}{e^{\alpha}+1}}}{e^{\alpha}+1}\right)^{n}
$$

as

$$
\sum_{j=0}^{\infty} \sum_{m=0}^{j}\left(\begin{array}{c}
j \\
m
\end{array}\right) \frac{\alpha^{n+j} U^{n+m}(-1)^{m} n^{j} e^{\alpha m}}{\left(e^{\alpha}+1\right)^{m}} .
$$

Put

$$
\Omega_{i, k, n, j, m}=\delta_{r, i}\left(\begin{array}{c}
i \\
k
\end{array}\right)\left(\frac{e^{\alpha}}{e^{\alpha}+1}\right)^{i-k} \frac{-k(-n)^{n-k-1}}{(n-k) !}\left(\begin{array}{c}
j \\
m
\end{array}\right) \frac{\alpha^{n+j}(-1)^{m} n^{j} e^{\alpha m}}{\left(e^{\alpha}+1\right)^{m}} .
$$

Thus

$$
\mu_{r}^{\prime}=\sum_{i=0}^{\infty} \sum_{k=0}^{i} \sum_{n=k}^{\infty} \sum_{j=0}^{\infty} \sum_{m=0}^{j} \Omega_{i, k, n, j, m} E\left[U^{i-k+n+m}\right] .
$$


Table 2: Some Ordinary Moments

\begin{tabular}{ccc}
\hline$r$ & $E\left(X^{r}\right)$ of $\operatorname{GAPAL}(0.5,0.3,-0.7)$ & $E\left(X^{r}\right)$ of $\operatorname{GAPAN}(0.5,0.3,-0.7)$ \\
\hline 1 & 0.3529384 & 0.4170033 \\
\hline 2 & 0.40257 & 0.2579811 \\
\hline 3 & 0.3339925 & 0.1806048 \\
\hline 4 & 0.5525787 & 0.1449196 \\
\hline 5 & 0.7012824 & 0.1270076 \\
\hline 6 & 1.593864 & 0.1209279 \\
\hline 7 & 2.758131 & 0.1229065 \\
\hline 8 & 8.166354 & 0.1325113 \\
\hline 9 & 8.166354 & 0.1503252 \\
\hline \hline
\end{tabular}

\subsection{Renyi Entropy}

Lemma 5.3. Let $f(x)$ denote the PDF of GAPA-G, then for $\delta>0$, and $\delta \neq 1$, $f(x)^{\delta}$ can be expressed as

$$
\sum_{q=0}^{\infty} \sum_{m=0}^{q} \sum_{v=0}^{\delta} \sum_{l=0}^{v} \sum_{r, j=0}^{\infty} \Omega_{q, m, v, l, r, j} g(x)^{\delta} G(x)^{m+v-l+t+j}
$$

where $\Omega_{q, m, v, l, r, j}$ is defined as in the proof of the Lemma, $g(x)$ and $G(x)$ are the PDF and CDF associated with the baseline distribution.

Proof. By the power series representation for the exponential function and the binomial theorem, we can write

$$
e^{\alpha \delta(G(x)-1)}=\sum_{q=0}^{\infty} \sum_{m=0}^{q}\left(\begin{array}{c}
q \\
m
\end{array}\right) \frac{\alpha^{q} \delta^{q}(-1)^{q-m}}{q !} G(x)^{m} .
$$


We can also write

$$
\left(\alpha G(x)+e^{\alpha G(x)}+1\right)^{\delta}=\sum_{v=0}^{\delta} \sum_{l=0}^{v}\left(\begin{array}{l}
\delta \\
v
\end{array}\right)\left(\begin{array}{l}
v \\
l
\end{array}\right) \frac{\alpha^{v-l+t} l^{t}}{t !} G(x)^{v-l+t} .
$$

On the other hand by the negative binomial series and the power series representation for the exponential function, we can write

$$
\left(e^{\alpha G(x)}+1\right)^{-2 \delta}=\sum_{r=0}^{\infty} \sum_{j=0}^{\infty} \frac{(-1)^{r}(r \alpha)^{j}}{j !}\left(\begin{array}{c}
2 \delta+r-1 \\
r
\end{array}\right) G(x)^{j} .
$$

Put

$\Omega_{q, m, v, l, r, j}=\left(\begin{array}{c}q \\ m\end{array}\right) \frac{\alpha^{q} \delta^{q}(-1)^{q-m}}{q !}\left(\begin{array}{l}\delta \\ v\end{array}\right)\left(\begin{array}{l}v \\ l\end{array}\right) \frac{\alpha^{v-l+t} l^{t}}{t !} \frac{(-1)^{r}(r \alpha)^{j}}{j !}\left(\begin{array}{c}2 \delta+r-1 \\ r\end{array}\right)\left(e^{\alpha}+1\right)^{\delta}$.

Thus,

$$
f(x)^{\delta}=\sum_{q=0}^{\infty} \sum_{m=0}^{q} \sum_{v=0}^{\delta} \sum_{l=0}^{v} \sum_{r, j=0}^{\infty} \Omega_{q, m, v, l, r, j} g(x)^{\delta} G(x)^{m+v-l+t+j}
$$

Let $X$ be a random variable with $\operatorname{PDF} f(x)$. By definition, the Renyi entropy [19] is defined as

$$
I_{R}(\delta)=\frac{1}{1-\delta} \log \left(\int_{-\infty}^{\infty} f^{\delta}(x) d x\right)
$$

where $\delta>0$, and $\delta \neq 1$. From the above Lemma we have the following

Theorem 5.4. The Renyi entropy of GAPA-G can be expressed as

$$
I_{R}(\delta)=\frac{1}{1-\delta} \log \left(\sum_{q=0}^{\infty} \sum_{m=0}^{q} \sum_{v=0}^{\delta} \sum_{l=0}^{v} \sum_{r, j=0}^{\infty} \Omega_{q, m, v, l, r, j} \int_{-\infty}^{\infty} g(x)^{\delta} G(x)^{m+v-l+t+j} d x\right)
$$

where $\delta>0$, and $\delta \neq 1$, and $g(x)$ and $G(x)$ are the PDF and CDF associated with the baseline distribution, $\Omega_{q, m, v, l, r, j}$ is defined as in the proof of the previous Lemma. 
For computational purposes if we let $u=F(x)$, then $d u=f(x) d x$ and $x=F^{-1}(u)$, thus the Renyi entropy can be expressed as

$$
I_{R}(\delta)=\frac{1}{1-\delta} \log \left(\int_{0}^{1}\left\{f\left(F^{-1}(u)\right)\right\}^{\delta-1} d u\right) .
$$

Table 3: Some Values of the Renyi Entropy

\begin{tabular}{ccc}
\hline & & \\
\hline$\delta$ & $I_{R}(\delta)$ of $\operatorname{GAPAL}(0.5,0.3,-0.7)$ & $I_{R}(\delta)$ of $\operatorname{GAPAN}(0.5,0.3,-0.7)$ \\
\hline 2 & 0.5479701 & 0.01703235 \\
\hline 3 & 0.4547242 & -0.05900715 \\
\hline 4 & 0.4002665 & -0.104941 \\
\hline 5 & 0.3638333 & -0.1363022 \\
\hline 6 & 0.3374348 & -0.1593408 \\
\hline 7 & 0.3172708 & -0.1771166 \\
\hline 8 & 0.3012786 & -0.1913245 \\
\hline 9 & 0.2882322 & -0.2029872 \\
\hline 10 & 0.2773527 & -0.2127625 \\
\hline \hline
\end{tabular}

\section{Parameter Estimation}

The method of maximum likelihood is used in this paper to estimate model parameters. Here we discuss this method for the GAPA- $G$ family of distributions. Suppose $x_{1}, x_{2}, \cdots, x_{n}$ is a random sample of size $n$ from the GAPA- $G$ family of distributions. It can be shown that the total log-likelihood function is given by

$$
\begin{aligned}
\ln L=\sum_{i=1}^{n}\{\ln (g(x ; \xi))+ & \ln \left(\left(e^{\alpha}+1\right)\right)+\ln \left(e^{\alpha(G(x ; \xi)-1)}\right) \\
& \left.+\ln \left(\alpha G(x ; \xi)+e^{\alpha G(x ; \xi)}+1\right)-\ln \left(\left(e^{\alpha G(x ; \xi)}+1\right)^{2}\right)\right\}
\end{aligned}
$$


where $\xi$ is a vector of parameters associated with the baseline distribution, and $\alpha \in \mathbb{R}$. Partial differentiation of the total log-likelihood function with respect to model parameters gives the following as the score functions

$$
\begin{aligned}
& \frac{\partial \ln L}{\partial \alpha}=\sum_{i=1}^{n}\left\{G(x ; \xi)+\frac{G(x ; \xi)+G(x ; \xi) e^{\alpha G(x ; \xi)}}{\alpha G(x ; \xi)+e^{\alpha G(x ; \xi)}+1}+\frac{2 G(x ; \xi) e^{\alpha G(x ; \xi)}}{e^{\alpha G(x ; \xi)}+1}\right\} \\
& \frac{\partial \ln L}{\partial \xi} \\
= & \sum_{i=1}^{n}\left\{\frac{\frac{\partial g(x ; \xi)}{\partial \xi}}{g(x ; \xi)}+a g(x ; \xi)+\frac{\alpha+\alpha e^{\alpha G(x ; \xi)}}{1+e^{\alpha G(x ; \xi)}+\alpha G(x ; \xi)} g(x ; \xi)+\frac{2 \alpha e^{\alpha G(x ; \xi)}}{e^{\alpha G(x ; \xi)}+1} g(x ; \xi)\right\} .
\end{aligned}
$$

Equating the score functions to zero and numerically solving the system of equations using techniques such as the quasi Newton-Raphson method, gives the maximum likelihood estimates for the model parameters. Let $\triangle=(\alpha ; \xi)$, for the purposes of constructing confidence intervals for the parameters in the GAPA- $G$ family of distributions, the observed information matrix, call it $J(\triangle)$, can be used due to the complex nature of the expected information matrix. The observed information matrix is given by

$$
J(\triangle)=-\left[\begin{array}{ll}
\frac{\partial^{2} \ln L}{\partial^{2} \alpha} & \frac{\partial^{2} \ln L}{\partial \alpha \partial \xi} \\
& \frac{\partial^{2} \operatorname{lnL}}{\partial^{2} \xi}
\end{array}\right] .
$$

When the usual regularity conditions are satisfied and that the parameters are within the interior of the parameter space, but not on the boundary, the distribution of $\sqrt{n}(\widehat{\triangle}-\triangle)$ converges to the multivariate normal distribution $N_{p+1}\left(0, I^{-1}(\triangle)\right)$, where $I(\triangle)$ is the expected information matrix, and it is assumed that $\xi=\left(\xi_{1}, \cdots, \xi_{p}\right)$. The asymptotic behavior remains valid when $I(\triangle)$ is replaced by the observed information matrix evaluated at $J(\widehat{\triangle})$. The asymptotic multivariate normal distribution $N_{p+1}\left(0, J^{-1}(\widehat{\triangle})\right)$ is a very useful tool for constructing an approximate $100(1-\psi) \%$ two-sided confidence intervals for the model parameters, where $\psi$ is the significance level. 


\section{Monte Carlo Simulation Study}

In this section we show that the method of maximum likelihood is adequate in estimating the parameters in the GAPA- $G$ distribution. For this, a Monte Carlo simulation study is carried out to assess the performance of the estimation method in the Gull Alpha Power Ampadu-Logisitic(GAPAL) sub-model. Samples of sizes 200, 400, 500, and 700, are drawn from the GAPAL distribution, and the samples have been drawn for the following set of parameters

(a) Set I: $(a, b)=(0.3,0.5)$ with fixed $c=0.7$

(b) $\underline{\text { Set II: }}(a, b)=(0.5,0.3)$ with fixed $c=-0.7$

The maximum likelihood estimators for the parameters $a$ and $b$ are obtained. The procedure has been repeated 400 times, and the mean and standard deviation for the estimates are computed, and the results are summarized in Table 4-6 below for each of sets I, and II, respectively, considered above

Table 4: Result of Simulation Study for Set I

\begin{tabular}{ccc}
\hline & \multicolumn{2}{c}{ Parameter $a$} \\
\hline Sample Size & Average Estimate & Standard Deviation \\
\hline 200 & 0.3005039 & 0.0637363 \\
\hline 400 & 0.2980213 & 0.04496851 \\
\hline 500 & 0.2962025 & 0.04042201 \\
\hline 700 & 0.2972835 & 0.02988517 \\
\hline \multicolumn{2}{c}{ Parameter $b$} \\
\hline Sample Size & Average Estimate & Standard Deviation \\
\hline 200 & 0.4977024 & 0.03006081 \\
\hline 400 & 0.499002 & 0.01985341 \\
\hline 500 & 0.4991902 & 0.01952368 \\
\hline 700 & 0.5000881 & 0.01566091 \\
\hline
\end{tabular}


From the table above, we find that the simulated estimates are close to the true values of the parameters and hence the estimation method is adequate. We have also observed that the estimated standard deviation consistently decrease with increasing sample size as can been seen by plotting the standard deviation against the sample size.

Table 5: Result of Simulation Study for Set II

\begin{tabular}{ccc}
\hline & \multicolumn{2}{c}{ Parameter $a$} \\
\hline Sample Size & Average Estimate & Standard Deviation \\
\hline 200 & 0.4951149 & 0.03788335 \\
\hline 400 & 0.4995736 & 0.02690994 \\
\hline 500 & 0.498642 & 0.02249279 \\
\hline 700 & 0.4966463 & 0.01937255 \\
\hline \multicolumn{2}{c}{ Parameter $b$} \\
\hline Sample Size & Average Estimate & Standard Deviation \\
\hline 200 & 0.298307 & 0.01899545 \\
\hline 400 & 0.2996662 & 0.01201761 \\
\hline 500 & 0.2997458 & 0.01140064 \\
\hline 700 & 0.2993985 & 0.009014662 \\
\hline
\end{tabular}

From the table above, we find that the simulated estimates are close to the true values of the parameters and hence the estimation method is adequate. We have also observed that the estimated standard deviation consistently decrease with increasing sample size as can been seen by plotting the standard deviation against the sample size.

Overall the simulation study conducted, indicated that using the method of maximum likelihood in estimating model parameters is adequate. 


\section{Applications}

Here we demonstrate usefulness of the new family to the breaking stress of carbon fibers data, Table 2 [20]. The PDF's and CDF's of the first submodel that we consider is already given in Section 4 . The second submodel has PDF given by

$$
=\frac{\left(e^{c}+1\right)\left(\operatorname{erfc}\left(\frac{a-x}{\sqrt{2} b}\right)+2 e^{\frac{1}{2} \operatorname{cerfc}\left(\frac{a-x}{\sqrt{2} b}\right)}+2\right) \exp \left(\frac{1}{2}\left(-\frac{(a-x)^{2}}{b^{2}}+\operatorname{erfc}\left(\frac{a-x}{\sqrt{2} b}\right)-2 c\right)\right)}{2 \sqrt{2 \pi} b\left(e^{\frac{1}{2} \operatorname{cerfc}\left(\frac{a-x}{\sqrt{2} b}\right)}+1\right)^{2}}
$$

and CDF given by

$$
G(x ; a, b, c)=\frac{\left(e^{-c}+1\right) \operatorname{erfc}\left(\frac{a-x}{\sqrt{2} b}\right)}{2\left(e^{-\frac{1}{2} \operatorname{cerfc}\left(\frac{a-x}{\sqrt{2} b}\right)}+1\right)}
$$

where $x, a, c \in \mathbb{R}, b>0$, and erfc gives the complementary error function. We call the distribution with the above PDF and CDF the Gull Alpha Power Ampadu-Normal family. We write

$$
J K \sim G A P A N(a, b, c)
$$

if $J K$ is a Gull Alpha Power Ampadu-Normal random variable. 


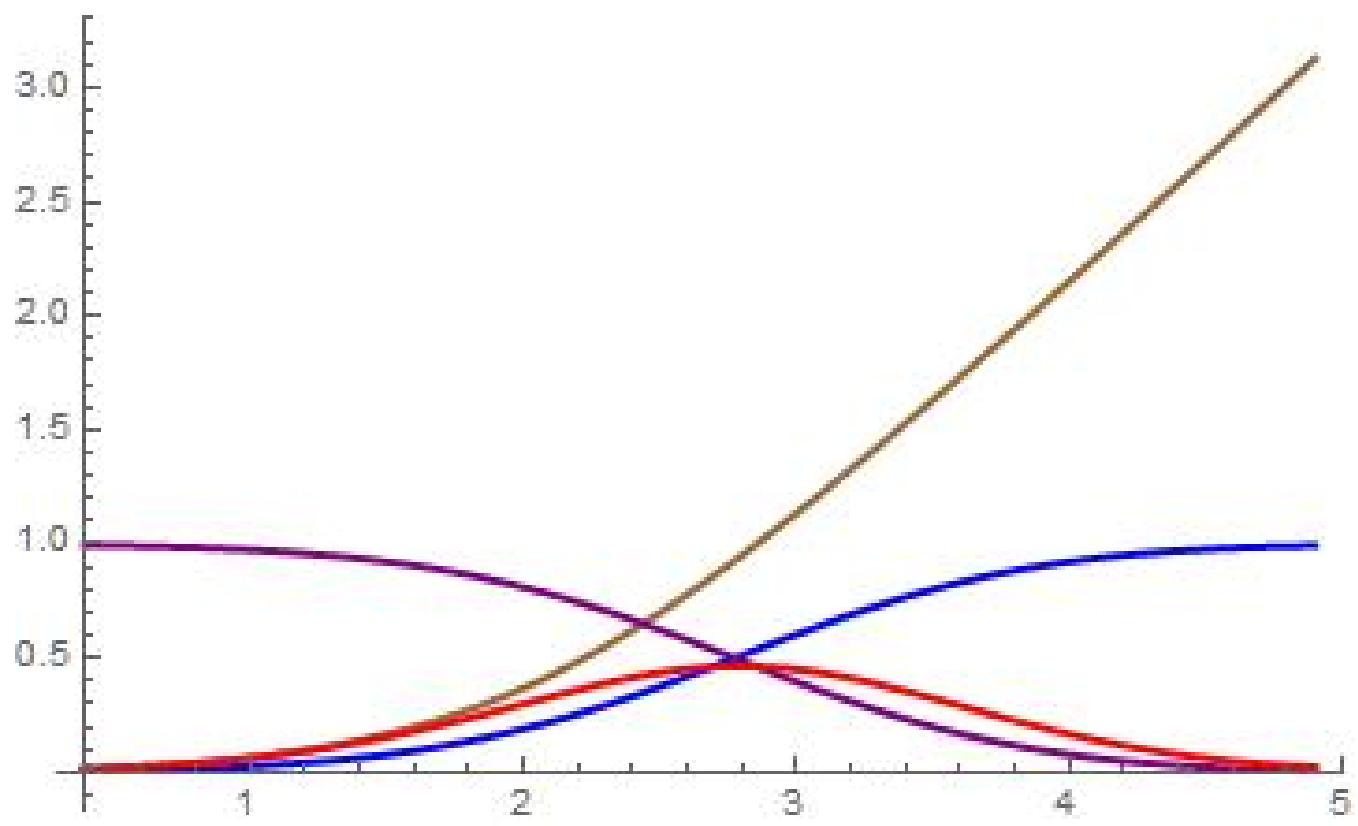

Figure 2: The CDF (blue), PDF(red), SF(purple), and HF (brown) of GAPAN $(2.59065,0.91193,1.46801)$.

Using the $\mathrm{R}$ software, we report below in Table 3, the estimates for the parameters in each of the two distributions alongside their standard errors.

Table 6: Estimates for the parameter of fitted distribution (c fixed as in the simulations)

\begin{tabular}{cccc}
\hline Distribution & Parameters & Estimates & Standard error \\
\hline GAPAL (with c fixed at -1.01248) & $\hat{a}$ & 3.2080931 & 0.10914232 \\
& $\hat{b}$ & 0.541204 & 0.05741692 \\
\hline GAPAN (with c fixed at 1.46801) & $\hat{a}$ & 2.590757 & 0.10990048 \\
& $\hat{b}$ & 0.911905 & 0.08158458 \\
\hline
\end{tabular}

The fitted CDF's to the carbon fibers data using the above table are shown below 

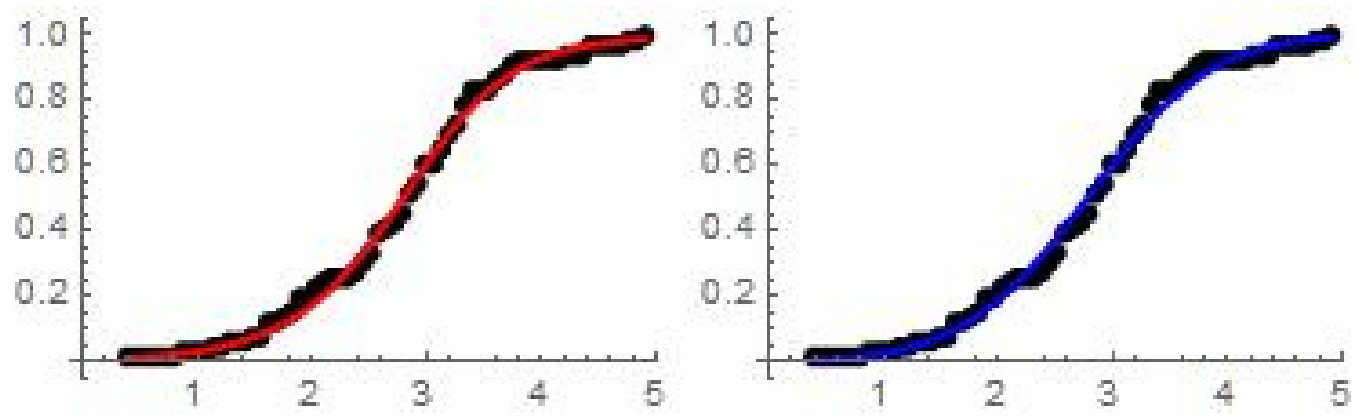

Figure 3: GAPAL (red), and GAPAN(blue) fitted to the empirical distribution of Table 2 [20].

and the fitted PDF's are shown below
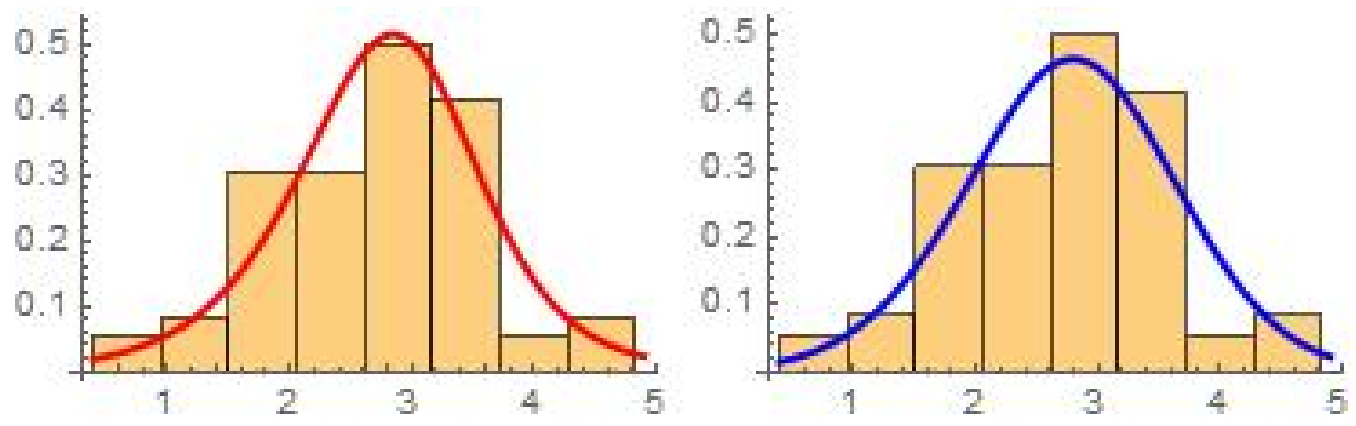

Figure 4: GAPAL (red), and GAPAN(blue) fitted to the histogram of Table 2 20].

The measures of goodness of fit we consider include Bayesian information criterion (BIC), negative Log-Likelihood, Cramer von-Misses (W), Anderson Darling (A), KS (Kolmogorov Smirnov), AIC (Akaikes Information Criterion), CAIC (Consistent Akaikes Information Criterion), and HQIC (Hannan-Quinn information criterion), and they are reported in Table 4 below. Whilst it appears from the fits above, that all the distributions are competitive in fitting the breaking 
stress of carbon fibers data, Table 4 reveals that the GAPAL distribution is most compatible with this data set, and hence can be considered the best in this instance.

Table 7: Goodness of fit measures

\begin{tabular}{ccc}
\hline & & \\
\hline & GAPAL & GAPAN \\
\hline $\mathrm{W}$ & 0.03062323 & 0.06657812 \\
\hline $\mathrm{A}$ & 0.2411352 & 0.3782608 \\
\hline KS statistic & 0.30768 & 0.067507 \\
\hline KS p-value & $7.485 \mathrm{e}-06$ & 0.9244 \\
\hline AIC & 174.4603 & 174.6588 \\
\hline CAIC & 174.6508 & 174.8492 \\
\hline BIC & 178.8396 & 179.0381 \\
\hline HQIC & 176.1908 & 176.3892 \\
\hline -Log(likelihood) & 85.23014 & 85.31938 \\
\hline \hline
\end{tabular}

\section{A Characterization Theorem}

It is well known that the hazard function, $h_{F}$, of a twice differentiable function, $F$, satisfies the first order differential equation

$$
\frac{f^{\prime}(x)}{f(x)}=\frac{h_{F}^{\prime}(x)}{h_{F}(x)}-h_{F}(x) .
$$

In this section we present a Kumaraswamy-GAPA- $G$ type distribution. The result here is inspired by [21]. First let us introduce the following

Definition 9.1. We say a random variable $X$ follows a Kumaraswamy- $G$ type distribution if its $\mathrm{CDF}$ is given by

$$
F(x ; \xi)=1-(1-G(x ; \xi))^{2}
$$


where $G$ is some baseline distribution, $x \in \operatorname{Supp}(G)$, and $\xi$ is a vector of parameters in the baseline distribution whose support depends on $G$.

Remark 9.2. Note that if we take $\lambda=1$ and $\varphi=2$ in equation (1) of [22], then we get the CDF in the above definition.

The PDF of the Kumaraswamy- $G$ type distribution is given by

$$
f(x ; \xi)=2 g(x ; \xi)(1-G(x ; \xi))
$$

where $g$ is the PDF of the baseline distribution. Clearly the hazard rate function of the Kumaraswamy- $G$ type distribution is given by

$$
h(x ; \xi)=\frac{2 g(x ; \xi)}{(1-G(x ; \xi))} .
$$

Theorem 9.3. Let $X: \Omega \mapsto \mathbb{R}$ be a continuous random variable. The PDF of $X$ is

$$
2 g(x ; \xi)(1-G(x ; \xi))
$$

for some baseline distribution with PDF $g$ and $C D F G$ if and only if its hazard rate function $h(x)$ satisfies the following differential equation

$$
h^{\prime}(x)-\frac{g^{\prime}(x)}{g(x)} h(x)=\frac{2 g(x)^{2}}{(1-G(x))^{2}}
$$

with boundary condition $h(0)=2 g(0)$.

Proof. If $X$ has PDF as stated in the theorem, then the differential equation as stated holds. Now if the stated differential equation holds, then

$$
\frac{d}{d x}\left\{g(x)^{-1} h(x)\right\}=2 \frac{d}{d x}\left\{(1-G(x))^{-1}\right\}
$$

which implies

$$
h(x)=\frac{2 g(x)}{1-G(x)}
$$

which is the hazard rate function of the Kumaraswamy- $G$ type distribution.

Clearly, a characterization of the Kumaraswamy-GAPA- $G$ type distribution. is obtained from the above theorem by letting the baseline PDF be given as in Section 3.2, and letting the baseline CDF be given as in Section 3.1. 


\section{Further Recommendation}

As a further recommendation we suggest obtaining some properties and applications of a so-called Type II Gull Alpha Power Transform family of distributions. We leave the reader with the following. Obiviously, the PDF can be obtained by differentiating the CDF below

Definition 10.1. A random variable $X$ will be called Type II Gull Alpha Power if its CDF can be expressed as

$$
\frac{\left(e^{\alpha}+1\right) F(x ; \xi)}{e^{\alpha F(x ; \xi)^{2}}+1}
$$

where $\alpha \in \mathbb{R}, \xi$ is a vector of parameters in the baseline distribution $F$, and $x \in \operatorname{Supp}(F)$.

\section{References}

[1] M. Ijaz, S. M. Asim, Alamgir, M. Farooq, S. A. Khan and S. Manzoor, A Gull Alpha Power Weibull distribution with applications to real and simulated data, PLoS ONE 15(6) (2020), e0233080. https://doi.org/10.1371/journal.pone.0233080

[2] Clement Boateng Ampadu, The Ampadu-G family of distributions with application to the T-X(W) class of distributions, Annal. Biostat. E Biomed. Appl. 1(4): 2019. ABBA.MS.ID.000518. https://doi.org/10.33552/ABBA.2019.01.000518

[3] A. Mahdavi and D. Kundu, A new method for generating distributions with an application to exponential distribution, Communications in Statistics - Theory and Methods 46 (2017), 6543-6557. https://doi.org/10.1080/03610926.2015.1130839

[4] Zubair Ahmad, Muhammad Ilyas and G. G. Hamedani, The extended alpha power transformed family of distributions: properties and applications, Journal of Data Science 17(4) (2019), 726-741.

[5] I. Elbatal, Zubair Ahmad, M. Elgarhy and Abdullah M. Almarashi, A new alpha power transformed family of distributions: properties and applications to the Weibull model, J. Nonlinear Sci. Appl. 12 (2019), 1-20.

https://doi.org/10.22436/jnsa.012.01.01 
[6] Zubair Ahmad, M. Elgarhy and Nasir Abbas, A new extended alpha power transformed family of distributions: properties and applications, Journal of Statistical Modelling: Theory and Applications 1(2) (2018), 13-28.

[7] Amal S. Hassan, Rokaya E. Mohamd, M. Elgarhy and Aisha Fayomi, Alpha power transformed extended exponential distribution: properties and applications, $J$. Nonlinear Sci. Appl. 12 (2019), 239-251.

https://doi.org/10.22436/jnsa.012.04.05

[8] Mohamed E. Mead, Gauss M. Cordeiro, Ahmed Z. Afify and Hazem Al-Mofleh, The alpha power transformation family: properties and applications, Pak. J. Stat. Oper. Res. XV(III) (2019), 525-545. https://doi.org/10.18187/pjsor.v15i3.2969

[9] Suleman Nasiru, Peter N. Mwita and Oscar Ngesa, Alpha power transformed Frechet distribution, Appl. Math. Inf. Sci. 13(1) (2019), 129-141.

https://doi.org/10.18576/amis/130117

[10] Maha A. Aldahlan, Alpha power transformed log-logistic distribution with application to breaking stress data, Advances in Mathematical Physics 2020, Article ID 2193787, 9 pp. https://doi.org/10.1155/2020/2193787

[11] Ceren Unal, Selen Cakmakyapan and Gamze Ozel, Alpha Power Inverted Exponential Distribution: Properties and Application, Gazi University Journal of Science 31(3) (2018), 954-965.

[12] Clement Boateng Ampadu and Abdulzeid Yen Anafo, Maximum likelihood estimation in an Alpha-Power, Annal. Biostat. $\&$ Biomed. Appl. 2(3) (2019), ABBA.MS.ID.000541. https://doi.org/10.33552/ABBA.2019.02.000541

[13] Malek Fathizadeh, Abbas Mahdavi and Leila Jabbari, Alpha-power power distribution, J. Biostat. Epidemiol. 4(3) (2018), 129-135.

[14] A. S. Malik and S. P. Ahmad, Transmuted Alpha Power Inverse Rayleigh Distribution: Properties and Application, J. Sci. Res. 11(2) (2019), 185-194. https://doi.org/10.3329/jsr.v11i2.38844

[15] S. Ihtisham, A. Khalil, S. Manzoor, S.A. Khan and A. Ali, Alpha-Power Pareto distribution: Its properties and applications, PLoS ONE 14(6) (2019), e0218027. https://doi.org/10.1371/journal.pone.0218027 
[16] Amal S. Hassan, M. Elgarhy, Rokaya E. Mohamd and Sharifah Alrajhi, On the alpha power transformed power Lindley distribution, Journal of Probability and Statistics 2019, Article ID 8024769, 13 pp.

[17] Suleman Nasiru, Peter N. Mwita and Oscar Ngesa, Exponentiated generalized transformed-transformer family of distributions, Journal of Statistical and Econometric Methods 6(4) (2017), 1-17.

[18] I.S. Gradshteyn and I.M. Ryzhik, Tables of Integrals, Series and Products, Academic Press, New York, 2007.

[19] A. Renyi, On measures of entropy and information, Proceedings of the Fourth Berkeley Symposium on Mathematical Statistics and Probability 1 (1961), 547-561.

[20] Ayman Alzaatreh, Carl Lee and Felix Famoye, $T$-normal family of distributions: a new approach to generalize the normal distribution, Journal of Statistical Distributions and Applications 2014, 1:16.

https://doi.org/10.1186/2195-5832-1-16

[21] Haitham M. Yousof, Morad Alizadeh, S. M. A. Jahanshahi, Thiago G. Ramires, Indranil Ghosh and G.G. Hamedani, The transmuted Topp-Leone G family of distributions: theory, characterizations and applications, Journal of Data Science 15 (2017), 723-740.

[22] Marcelino A.R. de Pascoa, Edwin M.M. Ortega and Gauss M. Cordeiro, The Kumaraswamy generalized gamma distribution with application in survival analysis, Statistical Methodology 8 (2011) 411-433.

https://doi.org/10.1016/j.stamet.2011.04.001

This is an open access article distributed under the terms of the Creative Commons Attribution License (http://creativecommons.org/licenses/by/4.0/), which permits unrestricted, use, distribution and reproduction in any medium, or format for any purpose, even commercially provided the work is properly cited. 